\title{
Hipertensão arterial: uma abordagem direcionada aos efeitos do treinamento, mecanismos hipotensivos e respostas a programas de exercícios Arterial hypertension: effects of physical training, hypotensive mechanisms and applicability of exercise programs
}

\author{
Kalline Russo*, Walace Monteiro**
}

*Laboratório de Atividade Física e Promoção da Saúde - Universidade do Estado do Rio de Janeiro (LABSAU/UERJ), ** Laboratório de Atividade Física e Promoção da Saúde - Universidade do Estado do Rio de Janeiro (LABSAU/UERJ), Programa de Pós-graduação em Ciências da Atividade Física - Universidade Salgado de Oliveira (UNIVERSO)

\section{Resumo}

A Hipertensão Arterial (HA) é uma condição multifatorial, associada a uma pressão arterial (PA) maior ou igual a 140/90 $\mathrm{mmHg}$ ou ao uso de medicamentos anti-hipertensivos. No Brasil, estudos epidemiológicos estimam uma prevalência da $\mathrm{HA}$ em torno de $40 \%$ da populaçáo adulta com mais de 40 anos. Uma das principais formas de prevenção e tratamento da HA consiste nas modificaçóes do estilo de vida. Dentre as modificaçôes, destaca-se a prática regular de atividade física. Desta forma, os objetivos do presente artigo são: a) revisar os efeitos do treinamento físico na PA; b) apresentar os potenciais mecanismos hipotensivos como resposta ao exercício; c) apresentar e discutir as diferentes estratégias de atividades físicas envolvendo programas formais e náo formais de prescrição de exercício. O primeiro objetivo aborda os efeitos agudos e crônicos das diferentes formas de treinamento (aeróbio, de força e flexibilidade), assim como a influência das diferentes variáveis de prescrição nas respostas da PA. O segundo objetivo apresenta os principais mecanismos neuro-humorais e estruturais que tentam explicar a hipotensão como resposta à prática do exercício. Quanto ao enfoque do terceiro objetivo, são discutidas a aplicabilidade, vantagens e desvantagens de programas formais e não-formais de prescrição de exercícios.

Palavras-chave: hipertensão, exercício, hipotensão, fisiologia cardiovascular, atividade física.

\begin{abstract}
The arterial hypertension (HA) is a multifactorial condition associated with blood pressure (BP) equal or higher than 140/90 $\mathrm{mmHg}$ or with the anti-hypertensive drugs. Epidemiological data estimate that $40 \%$ of the Brazilian adult population are older than 40 yrs. One of the alternative interventions to prevent and treat HA is the regular physical activity. Thus the purposes of the present paper are: a) to review the effects of physical training on the $\mathrm{HA}$; b) to describe the main hypotensive mechanisms associated with the exercise; c) to present and discuss the different strategies to prescribe physical activities for hypertensive subjects, especially supervised and non-supervised programs. The first objective focuses the acute and chronic effects of different training programs (aerobic, strength and flexibility), as well the influence of the manipulation of different prescription variables on BP responses. The second purpose presents the neuro-humoral mechanisms that explain the hypotension as consequence of regular exercise. The third purpose discusses the applicability, advantages and limitations of supervised and non-supervised exercise programs.
\end{abstract}

Key-words: hypertension, exercise, hypotension, cardiovascular physiology, physical activity. 


\section{Introdução}

A Hipertensão Arterial (HA) é uma condição multifatorial, associada a uma pressão arterial (PA) maior ou igual a $140 / 90 \mathrm{mmHg}$ ou ao uso de medicamentos anti-hipertensivos $[1,2]$, apresentando dois estágios em virtude dos níveis de pressão sistólica e diastólica do indivíduo. Para adultos de dezoito anos ou mais, que náo estáo tomando medicamentos ou tenham doença aguda, esses estágios são caracterizados da seguinte maneira: o estágio 1, (PA sistólica de $140 \mathrm{mmHg}$ a $159 \mathrm{mmHg}$ e PA diastólica de $90 \mathrm{mmHg}$ a $99 \mathrm{mmHg}$ ) e o estágio 2 (PA sistólica $\geq 160 \mathrm{mmHg}$ e PA diastólica $\geq 100$ $\mathrm{mmhg}^{3}$. Somado a isso, devido à incidência duas vezes maior do risco de desenvolver HA em pessoas com PA sistólica de $120 \mathrm{mmHg}$ a $139 \mathrm{mmHg}$ e PA diastólica de $80 \mathrm{mmHg}$ a $89 \mathrm{mmHg}$, uma nova categoria foi adotada para pacientes que apresentam esses valores de PA: a pré-hipertensão. Esta categoria foi introduzida reconhecendo a relação entre a PA e o risco de doenças cardiovasculares, sinalizando ainda, a necessidade de crescimento de educação para a saúde.

No Brasil, estudos epidemiológicos estimam prevalência em torno de $40 \%$ da população adulta com mais de 40 anos. Mas como o diagnóstico clínico requer avaliações sucessivas em diferentes ocasióes, a prevalência estimada da HA pode reduzir-se em 30\% [4]. Além disso, a HA é um dos principais agravos à saúde no Brasil, pois eleva o custo médico-social, principalmente pelas suas complicações, como as doenças cérebro-vascular, arterial coronariana e vascular de extremidades, além das insuficiências cardíaca e renal [5].

Diante desse quadro, as estratégias de prevenção são direcionadas para prevenir o desenvolvimento da hipertensão, ou a mudança de estágio da doença, como também o agravamento das condiçôes de saúde do paciente por complicaçóes facilitadas pela pressão arterial elevada. Autores como Appel et al. [6] e He, Whelton [7], destacam que as modificações comportamentais reduzem a morbi-mortalidade e o ônus causado pela HA e doenças a ela relacionadas, pois, incidem na redução de outros fatores de riscos para doenças cardiovasculares. Neste contexto, a adoção de um estilo de vida saudável é essencial na prevenção da HA. Dentre as modificações preconizadas no estilo de vida, destaca-se a prática sistemática de atividade física [8,9]. Algumas modificaçōes no estilo de vida apresentam custo mínimo, o que viabiliza sua realização em grande escala. Por essas razóes, algumas entidades como a Organização Mundial da Saúde, a Sociedade Européia de Hipertensão e o Programa Nacional de Educação para Pressão Arterial Elevada, recomendam a atividade física regular para prevenção e tratamento da HA [8].

Desta forma, os objetivos do presente artigo são: a) revisar os efeitos do treinamento físico na PA; b) apresentar os potenciais mecanismos hipotensivos como resposta ao exercício; c) apresentar e discutir as diferentes estratégias de atividades físicas envolvendo programas formais e não formais de prescrição de exercício.

\section{Exercício físico como estratégia de prevenção e intervenção}

Para facilitar o encadeamento de idéias, optamos por descrever esta sessão nos seguintes tópicos: exercício físico e seus efeitos agudos e crônicos na PA e aspectos metodológicos da prescrição de exercício.

\section{Efeitos agudos e crônicos do exercício na PA}

Como resposta aguda ao exercício aeróbio (EA), uma hipotensão pós-exercício tem sido observada. Essa ocorre em normotensos, hipertensos, adultos jovens e idosos, com a maior redução nos indivíduos com HÁ [8]. Além disso, estudos analisando os efeitos agudos do EA sobre a PA verificaram que as atividades dinâmicas reduzem a PA em pessoas com hipertensão, por uma maior porção de horas durante o dia [10]. Quanto aos efeitos da duração do esforço na PA, alguns estudos têm observado que a duração do exercício determina a magnitude e a duração da hipotensão pós exercício [11]. Esta hipotensão tem reduzido, em média, a PAS de 18 a $20 \mathrm{mmHg}$ e a PAD de 7 a $9 \mathrm{~mm} \mathrm{Hg}$, em indivíduos com hipertensão. Nos normotensos, os efeitos reportados variam de 8 a $10 \mathrm{mmHg}$ e 3 a $5 \mathrm{~mm} \mathrm{Hg}$, nas PAS e PAD, respectivamente, podendo persistir até 13 horas após a sessão de treinamento [12].

Em relação às respostas crônicas ao $\mathrm{EA}$, foi destacado que sua prática regular pode reduzir a pressão sistólica/diastólica em $3 / 2 \mathrm{mmHg}$ em adultos normotensos [13]. No que diz respeito à redução da $\mathrm{PA}$ em hipertensos, essa redução pode chegar a $6 / 7 \mathrm{mmHg}$, sendo a queda proporcional a PA inicial [14]. Como visto, os efeitos agudos na PA tendem a apresentar uma maior magnitude de redução, quando comparados aos efeitos crônicos ao esforço.

Paffenberger et al. [15] reportaram que exercícios classificados como vigorosos, independentemente do tipo, continuado por anos após a faculdade, protege contra futura hipertensão. Haapanen et al. [16] observaram que o total de atividade física, também independentemente do tipo somada com a intensidade do esforço, é inversamente associado com o risco de futura HA em homens de meia idade. Hayashi et al. [17] em estudo com homens japoneses, reportaram que a duração da caminhada para o trabalho, adicionada à realização de atividade física no tempo livre, foi significativamente associada com a redução do risco de hipertensão. Em contraste, nenhum dos estudos em mulheres observou significativas e independentes relaçôes entre o nível de atividade física e o risco para desenvolver a HÁ $[16,18,19]$. É importante observar, que características diferentes na PA em relação ao gênero podem ter influenciado nos resultados das pesquisas realizadas entre homens e mulheres. Segundo Hayes [20], diferenças em relação ao gênero vêm sendo notadas na fisiologia, genética $e$ benefícios no tratamento de $\mathrm{HA}$ em estudos que incluem mulheres. Legato [21] verificou que mulheres podem tolerar a HA 
melhor do que homens. Outro fator mais bem tolerado pelas mulheres é a microalbuminuria, interpretada diferentemente dos homens. A microalbuminuria representa um dos maiores fatores de risco para doenças cardiovasculares, associado com maior risco de mortalidade para hipertensos e principalmente diabéticos [22]. Por fim, algumas consideraçôes importantes que diferenciam o tratamento da mulher hipertensa, como o uso de contraceptivo oral, podem precipitar ou acentuar o problema, além das alteraçóes hormonais decorrentes da menopausa [21].

Em relaçáo aos estudos dos efeitos crônicos do EA em populaçóes da raça negra, poucos são os experimentos que investigaram os efeitos do exercício na PA. Parece ser aceito que a atividade física náo foi associada com a incidência de HA em estudos envolvendo negros [19]. As respostas da PA em população negra também diferem de homens de população branca, sendo encontrados valores de PA superiores em negros. Além disso, uma maior prevalência de HA e de complicaçôes cardiovasculares são encontrados em homens negros, em comparação com os brancos [23].

Quanto à influência do nível de condicionamento e a incidência de HA, Blair et al. [24], depois de controlar fatores como idade, gênero, índice de massa corporal e PA, reportaram que os indivíduos com baixos graus de condicionamento físico têm um maior risco relativo para desenvolver HA, quando comparado com pessoas bem condicionadas. Assim, os estudos até agora reportaram que altos níveis de atividade física, associados ao bom condicionamento aeróbio, associamse com a redução da incidência de HA em homens brancos. Entretanto, são necessárias mais evidências em relação ao gênero e as características étnicas, para que se possam tecer conclusões mais definitivas sobre o assunto [8].

\section{Aspectos metodológicos da prescrição de exercí- cios e respostas na PA}

Nessa sessão serão discutidas as influências da freqüência semanal, duração, intensidade e tipo de treinamento (aeróbio, força e flexibilidade) na PA. Forjaz et al. [11], analisaram indivíduos que realizaram duas sessóes de exercício (25 e $45 \mathrm{~min}$ ) no cicloergômetro em $50 \% \mathrm{VO}_{2}$ máx, onde a PAS diminuiu significativamente no pós-exercício, sendo essa queda maior e mais prolongada após $45 \mathrm{~min}$ de exercício. Esses autores concluíram que o exercício com maior duração provoca hipotensão pós-exercício maior e mais prolongada. Posteriormente, através de uma meta-análise Fagard et al. [25], defenderam que uma freqüência de três a cinco dias por semana, com duração de 30 a 60 min por sessão e intensidade entre $40 \%$ a $50 \%$ do $\mathrm{VO}_{2}$ máx, parece ser efetivo para a redução da PA. Contudo, limitadas evidências também sugerem que sete sessões por semana podem ser mais efetivas do que 3 sessóes por semana [26]. Outros autores corroboram com a intensidade proposta por Fagard, e vão além, destacando que os exercícios com baixa e moderada intensidade parecem provocar maiores reduções da PA [27-29]. Halbert et al. [30] complementam essas informaçôes observando que, aumentando a intensidade do exercício para $70 \% \mathrm{VO}_{2}$ máx e aumentando a freqüência semanal acima de 3 vezes não tem nenhum impacto adicional na reduçáo da PA.

Um dos aspectos que podem ter influenciado nas diferenças entre os estudos, pode estar relacionado às diferentes populaçóes que compuseram as amostras dos estudos, envolvendo indivíduos com distintos graus de condicionamento, composição corporal, além das diferenças metodológicas nos treinamentos propostos. Apesar da divergência quanto à freqüência semanal que provocaria um maior efeito hipotensivo, parece ser lógico assumir que uma margem mínima situaria em três dias e uma margem ótima de treinamento deve ser de cinco a sete sessóes semanais. Obviamente, os aspectos clínicos do indivíduo bem como o risco de lesôes impostas pela atividade aeróbia praticada devem ser levados em conta na escolha da freqüência semanal. Além disso, a interação duração-intensidade do esforço também deve ser considerada.

No que concerne aos afeitos agudos do TF na PA, os resultados das pesquisas parecem ser convergentes. Farinatti e Assis [31] verificaram as respostas cardiovasculares durante exercícios de força e aeróbio em indivíduos saudáveis. Realizaram-se testes de força com 1RM, 6RM e 20RM na cadeira extensora e exercício aeróbio em cicloergômetro na intensidade de 75 a $80 \%$ da FC de reserva. Os dados de PAS mostraram uma elevação conforme o número de repetições foi aumentando nos testes de força, o mesmo ocorrendo em relação à PAD. Quanto à comparação das respostas de PA obtidas na atividade aeróbia e nos testes de força, com exceção do trabalho envolvendo 20RM, o trabalho aeróbio exibiu maiores respostas de PAD. Já a PAS foi superior na atividade aeróbia do que nos testes de força. Esses dados chamam a atençáo para o fato de que as respostas de PA tendem a ser menores no TF do que no treinamento aeróbio.

Quanto à influência do número de séries sobre as respostas da PA, Gotshall et al. [32] analisaram os efeitos de 3 séries consecutivas de 10 repetiçóes sobre a resposta da PA no exercício leg press. Em conclusão, quanto maior o número de séries maiores as respostas obtidas para PA. Mais recentemente, Polito et al.[33] verificaram o comportamento das respostas cardiovasculares durante 4 séries de 8 repetiçôes máximas (RM) na extensão unilateral do joelho, realizadas com $1 \mathrm{e}$ 2 minutos de recuperaçáo. No que concerne às respostas de $\mathrm{PA}$, verificou-se um efeito somativo do número de séries na PAS e PAD. Quanto à influência dos diferentes intervalos de recuperação, ao se adotar o período de 2 minutos, a PAS e $\mathrm{PAD}$ atingiram menores valores ao final de cada série de $8 \mathrm{RM}$, em relação aos valores obtidos com 1 minuto de intervalo. A influência do fracionamento das séries através da alternância de grupamentos musculares requisitados na realização dos exercícios também vem sendo estudada. Veloso et al. [34] verificaram as respostas cardiovasculares em diferentes situ- 
açóes de TF para flexão unilateral do quadril e abdução dos ombros até $90^{\circ}$. Os exercícios foram conduzidos de forma a manter o mesmo volume de treinamento, variando-se o número de repetiçóes contínuas. Os valores de PA revelaram-se significativamente maiores na forma contínua de execução, em comparação com a fracionada. Os autores concluíram que a forma contínua associou-se a maior sobrecarga cardiovascular, principalmente devido às respostas de pressão arterial.

A hipotensão pós-exercício com a prática do treinamento de força também vem sendo estudada. Em estudos realizados com jovens saudáveis e treinados Polito et al. [35] estudaram o efeito de duas seqüências de TF realizados sobre intensidades diferentes, mas com o mesmo volume de treinamento, sobre as respostas agudas tardias de PAS e PAD. Os autores chegaram as seguintes conclusóes: a) o TF exerceu o efeito hipotensivo sobre a PA, principalmente sobre a PAS; b) o declínio absoluto da PAS não foi influenciado pelas diferentes interaçôes de carga e repetiçóes; c) a magnitude das cargas tendeu a favorecer a duração da redução da PAS e d) o número de repetições teve maior repercussão sobre a $\mathrm{PAD}$ que sobre a PAS, mas por curto período de tempo. Posteriormente, Simão et al. [36] também verificaram os efeitos hipotensivos de diferentes formas de treinamento. Inicialmente os autores compararam os efeitos do trabalho conduzido com séries consecutivas para cada exercício realizado com $6 \mathrm{RM}$ e 12 repetiçóes com cargas correspondentes a 50\% de 6RM. Depois foram comparados os efeitos dos trabalhos de 6RM realizados em séries consecutivas com aqueles obtidos em 12 repetiçóes com cargas correspondentes a 50\% de 6RM, desta vez realizada em forma de circuito. Em conclusão, não foram verificadas diferenças na magnitude do efeito hipotensor nas diferentes formas de treinamento. Contudo, o efeito tendeu a perdurar mais no trabalho de maior intensidade (6RM) e circuito, em comparação ao trabalho de 12 repetiçóes. Outros estudos também verificaram hipotensão pós-exercício [37-39]. Em contrapartida, algumas investigaçôes verificaram apenas discreta hipotensão pós TF. Fisher et al. [40], estudaram mulheres normotensas e hipertensas. Após a realização de 15 repetiçóes em cinco exercícios conduzidos em circuito, com carga equivalente a 50\% de 1RM, verificou-se redução significativa somente na PAS. Outros estudos não puderam demonstrar efeito hipotensivo significativo após o exercício de força. Focht e Koltyn [41], por exemplo, observaram redução apenas na PAD 20 minutos após uma seqüência de exercícios realizada a 50\% de 1 RM. Os autores não verificaram alteraçóes no TF realizado na intensidade de trabalho equivalente a $80 \%$ de 1RM.

Em relação à resposta crônica do TF na PA de repouso, essa é uma questão que merece ser melhor investigada, pois os resultados das pesquisas são conflitantes. Apesar disso, algumas evidências iniciais sugerem que o TF, em certos casos, pode exercer um efeito hipotensivo na PA de repouso [42-44]. É importante destacar que mesmo que o efeito não seja estatisticamente significativo, reduçóes de $3 \mathrm{mmHg}$ na PAS pode ser interessante para reduzir o risco de possíveis complicaçóes cardiovasculares $[8,45]$. Em interessante meta-análise sobre os efeitos do TF na PA, Kelley e Kelley [45] concluíram que o TF pode ser eficaz na redução da PAS e PAD em adultos. Mesmo nos estudos que não demonstraram redução da $\mathrm{PA}$ com o TF, verificou-se que essa forma de treinamento não traz como efeito crônico um aumento da PA. Tal fato se mostra interessante para o aprimoramento da aptidão física em hipertensos, o que justifica sua prática por indivíduos com HA. Contudo, os autores supracitados alertaram que as pesquisas necessitam de maior controle envolvendo as amostras, seus estados clínicos, bem como as características que envolvem o controle farmacológico dos indivíduos. Além disso, pode-se acrescentar que as variaçôes das características metodológicas que regem a prescrição do exercício, também, merecem futuras investigaçôes para que possam tecer conclusôes mais consistentes. Em geral, as consideraçôes metodológicas para a prescrição do TF em hipertensos envolvem 8 a 12 exercícios, realizados com 1 a 2 séries, executadas com 8 a 12 repetiçóes 2 a 3 vezes por semana $[8,29,46]$.

No que diz respeito ao exercício de flexibilidade, não há evidências sobre possíveis influências desse treinamento na PA. Porém, como qualquer outro componente da aptidão física, a flexibilidade deve ser treinada, tendo em vista que é um dos fatores que podem afetar a autonomia do indivíduo para realizaçáo de atividades do cotidiano [47]. Este aspecto se torna ainda mais importante quando se trata de idosos, sedentários e obesos. Muitos estudos sugerem métodos específicos com essa finalidade. No entanto, podemos afirmar que ainda há muitas limitaçóes no conhecimento sobre o assunto. Ao contrário do treinamento aeróbio, a intensidade, duração e freqüência dos estímulos no treinamento da flexibilidade ainda estão longe de serem definidos [48].

\section{Mecanismos potenciais para redução da PA em resposta a atividade física}

Existem diferentes mecanismos que tentam explicar a hipotensão como resposta à prática do exercício. São eles os neuro-humorais e os estruturais.

Recentes estudos sugerem que a redução na PA depois de treinamento aeróbio é mediada pelo decréscimo do débito cardíaco e/ou da resistência periférica total. Porém, somente em alguns estudos com idosos, a hipotensão foi relacionada tanto por aumento da vasodilatação periférica quanto por diminuição no débito cardíaco [49]. Talvez, esse fato esteja relacionado às características da mostra, que tende a possuir uma FC mais baixa, devido ao envelhecimento. Todavia, uma redução no débito cardíaco não ocorre tipicamente depois de exercício crônico. Assim, o decréscimo na resistência periférica total aparece como mecanismo primário para reduzir a PA de repouso após treinamento [8].

Forjaz et al. [11] concordam com essa afirmação, e relatam que a redução na resistência vascular pode estar relacionada à vasodilatação provocada pelo exercício físico, tanto na 
musculatura ativa, como inativa. Essa redução da resistência vascular, após esforço, é mediada por adaptaçôes neuro-humorais e estruturais. O ACSM [8] destaca que as adaptaçóes neuro-humorais dizem respeito às adaptações no sistema nervoso simpático, sistema renina-angiotensina, bem como nas respostas vasculares. Já em relação às adaptaçôes estruturais, estudos como o de Laughlin et al. [50] sugerem que o treinamento modifica a estrutura vascular muscular, incluindo o remodelamento vascular como o aumento da flexibilidade, aumento da área de passagem do fluxo sangüíneo e/ou diâmetro das artérias e veias, e ainda a angiogênesis.

Elevada atividade do sistema nervoso simpático é um ponto importante observado na hipertensão essencial [51]. Alguns estudos demonstram que em sujeitos hipertensos a atividade nervo simpática é mais elevada quando comparada com sujeitos normotensos [18,52,53]. Somado a este fato, é importante observar que a atividade nervo simpática e a subseqüente liberação de norepinefrina (NE) media a vasoconstricção e aumenta a resistência vascular, podendo ser relacionada à maior resistência vascular observada em hipertensos. Apesar de limitadas evidências, estudos como os de Jennings et al. [54], Meredith et al. [55], e Urata et al. [56] observaram redução da quantidade de NE no plasma sangüíneo após treinamento aeróbio. Esta constatação foi relacionada ao decréscimo de sua liberação no plasma e não na sua remoção, sugerindo menor atividade do sistema nervoso simpático [55]. Sendo assim, menor quantidade de NE para as sinapses pode ser um mecanismo facilitador da redução da resistência vascular após treinamento, e ainda, outros efeitos associados com a inibição da produção simpática renal podem ser importantes no decréscimo da PA [8].

As adaptações vasculares também são prováveis contribuintes para diminuiçáo da PA após o treinamento. Alguns estudos vêm observando que o exercício altera as respostas vasculares para NE e para endotélio-1, que representam potentes vasoconstrictores. Um estudo realizado com ratos hipertensos espontâneos observou que exercícios crônicos desencadeiam um decréscimo da vasoconstricção por receptores $\alpha$-adrenérgicos [57]. Além disso, as respostas vasculares para estimulação dos receptores $\alpha$-adrenérgicos, pela $\mathrm{NE}$, são atenuadas depois do treinamento [58,59]. Somado a isso, Maeda et al. [60] reportaram que o treinamento também reduz os níveis de endotélio-1 em indivíduos hipertensos espontâneos. Se para agentes vasoconstrictores o exercício parece diminuir suas açôes, para agentes vasodilatadores como o óxido-nítrico, o exercício tem demonstrado um aumento da produção, promovendo a função vasodilatadora em sujeitos saudáveis [61].

Outro fator importante, associado com a HA, é a hiperinsulinimia e insulino resistência que vem sendo associado com a ativação do sistema nervoso simpático [62-64]. Devido ao exercício estimular a sensibilidade à insulina, o esforço também pode ser um importante mecanismo mediador da redução da atividade simpática e PA [65].
Adaptação neuro-humoral, também é encontrada no sistema renina-angiotensina. A angiotensina II é um poderoso vasoconstrictor e regulador do volume sangüíneo. Reduçôes na renina e angiotensina II com treinamento podem ser contribuintes para a redução da PA em normotensos [8]. Níveis reduzidos de renina e angiotensina II, após o treinamento, vem sendo reportados $[54,66]$. Contudo, em sujeitos hipertensos, os exercícios não reduzem consistentemente o plasma renal $[67,68]$ e os níveis de angiotensina II $[69,70]$. Assim, os estudos sugerem que o sistema renina-angiotensina II não contribui consideravelmente para a reduçáo da PA após o treinamento [8].

Devido à multifatoriedade dos mecanismos e redundantes sistemas que contribuem para a redução da PA, conclusóes definitivas sobre a hipotensão pós exercício se fazem difíceis. Contudo, mesmo sem conclusões definitivas, o fato é que, o exercício aeróbio desencadeia uma hipotensão pós-exercício, sendo esta de extrema importância no caso de pessoas com HA.

\section{Tipos de programas de exercício}

Pode-se dizer que a prescrição de atividades físicas para a prevenção e tratamento da hipertensão pode ser estruturada de acordo com duas vertentes metodológicas distintas: a) programas de atividades físicas formais ou intra-muros; b) programas de atividades físicas não formais ou extra-muros [71].

Os programas formais são aqueles onde existe um rígido acompanhamento dos aspectos metodológicos que regem a prescrição do exercício, como intensidade e duração do esforço. Neste tipo de programa, as variáveis clínicas e fisiológicas são mais controladas. Para que tal controle seja alcançado, é necessária a participação de um profissional especializado para acompanhar as sessóes de treinamento, sendo geralmente realizados em clínicas e hospitais. Os programas intramuros apresentam como vantagem a maior monitorização clínica e funcional durante as sessóes. Em contrapartida, tendem a provocar uma dependência dos indivíduos que a realizam. Já os programas não formais são aqueles onde a prescrição do exercício é organizada para levar o indivíduo a uma maior autonomia. Nesse tipo de programa, abre-se mão de um controle extremamente apurado em prol de uma maior possibilidade de realização das atividades por parte dos indivíduos. Os programas extramuros podem ser realizados em locais e horários que mais se ajustem as características do praticante. A metodologia de prescrição de exercícios é simples, de modo que o praticante tenha autonomia para realizar o controle do treinamento. Os programas não-formais envolvem recursos humanos e materiais reduzidos, podendo constituir uma interessante estratégia de saúde pública.

Sendo assim, é possível afirmar que os programas formais de exercícios seriam aqueles que deram origem à quase totalidade das pesquisas relacionadas à hipertensão e exercício. Contudo, apesar da vantagem de possuir maior ênfase no 
controle das atividades, geralmente a prática regular do exercício não é incorporada na vida dos indivíduos quando recebem alta. Em contrapartida, programas não formais, apesar do menor controle das atividades, podem favorecer a adesão dos indivíduos por períodos prolongados de tempo, demonstrando também um grande potencial a ser explorado para pesquisas.

Como destacado por Bar-Eli [72], atribuiçóes como liberdade, escolha e responsabilidade sobre a atividade aumentam o engajamento dos pacientes em programas de atividade física. Há também, freqüentes relatos que evidenciam ser a falta de tempo para a realização de exercícios regulares, um dos principais motivos para a não realização dos exercícios [73]. Programas que permitam certa flexibilidade na escolha do momento de suas realizaçôes mostram-se eficazes para uma maior adesão, tendo em vista, que em geral, os indivíduos têm uma precária organização do tempo [74].

Smith et al. [75] afirmam que a adesão tem se constituído em motivo de preocupação entre médicos, professores de educação física e outros profissionais da área de saúde. Cox et al. [76], por sua vez, observam que alguns estudos mostram que pacientes têm adesão aumentada quando realizam programas de atividades físicas de leve a moderada intensidade, ou seja, em uma faixa de conforto. Somado a isto, Farinatti [71] relata que as evidências de que a intensidade do exercício é um fator secundário para os efeitos esperados sobre a pressão arterial, em comparação com a duração e a regularidade de sua prática, abrem a possibilidade de que se abdique do controle fisiológico das atividades, em prol de outros objetivos como a adesão. Biddle e Mutrie [73] reforçam essa idéia, citando que uma ótima estratégia para uma adesão aumentada dos pacientes aos programas de treinamento é o automonitoramento.

Um interessante estudo sobre a influência de programas não formais na aptidão física, PA e variáveis bioquímicas em pacientes hipertensos foi realizado por Pinto et al. [77] ao investigar dois programas não-formais de atividade física os autores observaram que esses exercem efeitos positivos sobre a condição geral dos pacientes hipertensos. Isso foi observado principalmente no que diz respeito à composição corporal, em termos quantitativos e de distribuição regional. No que diz respeito à aptidão cardiorespiratória e $\mathrm{PA}$, foram pouco conclusivos, já que houve diferenças significativas no sentido de melhora, porém não houve um padrão de comportamento que desse suporte a esses possíveis efeitos.

Posteriormente, Farinatti et al. [48] observaram a influência de quatro meses de um programa domiciliar não-supervisionado (não-formais) de exercícios sobre a PA e aptidão física em adultos com hipertensão estágios I e II. Foram observados dois grupos: experimental e controle, compostos por indivíduos de ambos os sexos. O grupo experimental submeteu-se a um programa domiciliar de exercícios, com atividades fundamentalmente aeróbias $(60-80 \%$ da freqüência cardíaca máxima estimada para a idade, 30 minutos de caminhadas no mínimo três vezes por semana), além de exercícios de flexibilidade. Orientaçôes sobre a ficha de controle e variáveis intervenientes ao treinamento eram dadas a cada reavaliação. Os pacientes foram acompanhados por quatro meses, com reavaliaçôes que se dava a cada dois meses. Em conclusão, programas domiciliares não supervisionados de exercícios, mesmo em curto prazo, podem exercer efeito positivo sobre a pressão arterial e aptidão física de pacientes hipertensos.

Apesar de alguns estudos demonstrarem que é possível obter efeitos positivos com programas não-formais de exercícios, futuras pesquisas necessitam ser desenvolvidas para avaliar os efeitos dessas intervenções nas variáveis de aptidão física e PA de hipertensos. Um desafio para a saúde pública é fazer com que o exercício seja praticado em grande escala. Nesse sentido, programas não-formais por sua fácil aplicação e baixo custo, emergem como uma interessante estratégia de prevenção e tratamento da HA.

\section{$\overline{\text { Conclusão }}$}

A prática de exercícios constitui uma importante estratégia na prevenção e tratamento da HA. Ela é uma das principais modificaçôes de estilo de vida que contribui para reduzir diversos outros fatores de risco associados às doenças cardiovasculares. Além disso, níveis de condicionamento mais elevados estáo associados a uma menor incidência de HA.

Para que o exercício possa exercer seus efeitos positivos, sua prática deve ser regular. Entre as diferentes formas de exercícios, a atividade aeróbia é aquela que apresenta um maior efeito hipotensor. As evidências científicas sobre o efeito hipotensor da atividade aeróbia são mais consistentes do que aquelas obtidas para o TF. Porém, os resultados das pesquisas indicam que o TF deve ser incorporado como forma de aprimoramento da aptidão física relacionada à saúde, mesmo que seus efeitos hipotensivos na PA de repouso ainda sejam questionados. As características metodológicas que regem a prescrição do treinamento aeróbio para indivíduos hipertensos incluem: a) freqüência semanal: superior a 3 vezes, embora essa freqüência também se mostre suficiente para exercer efeitos hipotensivos; b) intensidade: 40 a $70 \%$ do $\mathrm{VO}_{2}$ máx; c) duração: de 30 a 60 minutos. Quanto ao TF, as considerações metodológicas para a prescrição em hipertensos devem envolver: a) número de exercícios: 8 a 12; b) número de séries e repetiçôes: 1 a 2 séries, executadas com 8 a 12 repetiçôes; c) freqüência: 2 a 3 vezes por semana. Aspectos como número de repetiçôes e cargas, intervalos entre séries, bem como a forma de execuçáo dos exercícios (fracionada ou contínua), influenciam nas respostas agudas da PA.

Os mecanismos hipotensivos ainda não estão bem estabelecidos, merecendo estudos adicionais para que se possam tecer inferências consistentes sobre o assunto. Os principais mecanismos de hipotensão como resposta à prática do exercício podem ser caracterizados como neuro-humorais e estruturais. As adaptaçóes neuro-humorais dizem respeito às adaptaçóes no sistema nervoso simpático, sistema reni- 
na-angiotensina, bem como nas respostas vasculares. As estruturais envolvem a modificação da estrutura vascular muscular, incluindo o remodelamento vascular como o aumento da flexibilidade, aumento da área de passagem do fluxo sangüíneo e/ou diâmetro das artérias e veias, e ainda a angiogênesis.

Por fim, cabe ressaltar que os programas de prescrição de exercícios podem ser caracterizados em formais ou intramuros e não-formais ou extramuros. Os programas formais apresentam como vantagem o maior controle das atividades prescritas e das respostas fisiológicas, o que pode ser interessante de acordo com o risco do indivíduo. Em contrapartida, programas com essas características, não tem se mostrados efetivos para manter a adesáo dos praticantes após a alta. Nos programas não-formais a prescrição é simples, existindo menor controle das atividades. Não é necessária a presença de um profissional especializado conduzindo todas as sessōes de treinamento. Uma das maiores vantagens desse tipo de programa é a autonomia que ele tende a gerar nos praticantes. Cabe ressaltar que náo existe a melhor forma de treinamento. Dependendo de aspectos como as características clínicas e funcionais, bem como as condições para a prática do exercício, a opção deve ser feita.

\section{Referências}

1. Van den Hoogen PC, Seidell JC, Menotti A, Kromhout D. Blood pressure and long-term coronary heart disease mortality in the Seven Countries study: implications for clinical practice and public health. Eur Heart J 2000;21:1639-42

2. Vasan RS, Larson MG, Leip EP et al. Impact of High-normal blood pressure on the risk of cardiovascular disease. $\mathrm{N}$ Engl J Med 2001;345:1291-97.

3. Joint National Committee on Detection, Evalution, and Treatment of High Blood Pressure. The seventh report of the Joint National Committee on Detection, Evalution, and Treatment of High Blood Pressure. (JNC VII). Arch Intern Med 2003.

4. Organização Mundial da Saúde. Organização Pan-Americana de Saúde. Avaliação do plano de reorganização da atenção à hipertensão arterial e ao diabetes mellitus no Brasil. Brasília: MS; 2004. 63 p. il. Color (Série C. Projetos, Programas e Relatórios).

5. Mion D, Machado CA, Gomes MAM et al. IV Diretrizes Brasileiras de Hipertensão Arterial. Arq Bras Cardiol 2004;82 (suplemento IV).

6. Appel LJ; Moore TJ; Obarzanek EA. Clinical trial of the effects of dietary patterns on blood pressure. New Eng J Med 1997;336:1117-1124.

7. He J, Whelton PK. Epidemiology and prevention of hipertension. Med Clin North Am 1997;5:1059-1077.

8. Pescatello LS, Barry A, Fagard RMD et al. Exercise and hypertension. Med Sci Sports Exerc 2004;36(3):533-553.

9. Kaplan et al. Task Force 5: Systemic Hypertension. J Am Coll Cardiol 2005; 45:1346-8.

10. Pescatello LS, Bairos L; Vanheest JL et al. Postexercise hypotension differs between white and black women. Am Heart J 2003;145:364-370.
11. Forjaz CLM, Santaella DF, Rezende LO, Barreto ACP, Negrão CE. Exercise duration determines the magnitude and duration of pos-exercise hypotension. Arq Bras Cardiol 1998;70:99-104.

12. Kenney MJ, Seals DR. Postexercise hypotension: key features, mechanisms, and clinical significance. Hypertens 1993;22:653664.

13. Mion D, Machado CA, Gomes MAM et al. IV Diretrizes Brasileiras de Hipertensão Arterial. Arq Bras Cardiol 2004; 82 (suplemento IV).

14. Kelley GA, Kelley KS, Tran ZV. Aerobic exercise and resting blood pressure: a meta-analytic review of randomized, controlled trials. Prev Cardiol 2001;4:73-80.

15. Paffenbarger Junior RS, Wing AL, Hyde RT, Jung DL. Physical activity and Incidence of hypertension in college Alumni. Am J Epidemiol 1983;117:245-257.

16. Haapanen NS, Miilunpalo I, Vuori PO, Pasanen M. Association of leisure time physical activity with the risk of coronary heart disease, hypertension and diabetes in middle-aged men and women. Int J Epidemiol 1997;26:739-747.

17. Hayashi TK, Tsumura C; Suematsu K; Okada S; Fujii, Endo G. Walking to work and the risk for hypertension in men: the Osaka health Survey. Ann Intern Med 1999;130:21-26.

18. Folsom AR, Prineas RJ, Kaye AS, Munger RG. Incidence of hypertension and stroke in relation to body fat distribution and other risk factors in older women. Siroke 1990;21:701-706.

19. Pereira MA, Folsom AR, Mcgoverns PG et al. Physical activity and incident hypertension in black and white adults: the Atherosclerosis Risk in Communities study. Prev Med 1999;28:304-312.

20. Hayes DL, Barold SS, Camm AJ, Goldschlager NF. Evolving indications for permanent cardiac pacing: an appraisal of the 1998 American College of Cardiology/American Heart Association Guidelines. Am J Cardiol 1998; 1;82(9):1082-6.

21. Legato MJ. Cardiovascular disease in women: gender-specific aspects of hipertension and the consequences of treatment. J Womens Health 1998;7(2):199-209.

22. Romundstad S, Holmen J, Hallan H, Kvenild K, Ellekjaer H. Microalbuminuria and all-cause mortality in treated hypertensive individuals: does sex matter? The Nord-Trondelag Health Study (HUNT), Norway. Circulation 2003;108(22):2783-9.

23. Kokkinos PF, Narayan P, Colleran JA et al. Effects of regular exercise on blood pressure and left ventricular hypertrophy in African-American men with severe hypertension. N Engl J Med 1995;333:1462-67.

24. Blair SM, Goodyear NN, Gibbons LW, Cooper KH. Physical fitness and incidence of hypertension in health normotensive man and women. JAMA 1984;252:487-90.

25. Fagard RH. Exercise characteristics and the blood pressure response to dynamic physical training. Med Sci Sports Exerc 2001;33(suppl):S484-92; discussion S 493-94.

26. Jennings GL, Deakin G, Korner P, Meredith I, King Well B, Nelson L. What is the dose-response relationship between exercise training and blood pressure? Ann Med 1991;23:313-18.

27. Hagberg JM, Goldring D, Ehsani AA et al. Effect of exercise training on the blood pressure and hemodynamic features of hypertension adolescents. Am J Cardiol 1983;52:763-768.

28. Cleroux J, Feldman RD, Petrella RJ. Lifestile modifications to prevent and control hypertension. CMAJ1999;160(9):521528. 
29. American college of sports medicine. Guidelines of exercise testing and exercise prescription. $4 \mathrm{a}$ ed. Philadelphia: Lea $\&$ Febiger; 2000.

30. Halbert JA, Silagy CA, Finucane P, Withers RT, Hamdorf PA, Andrews GR. The effectiveness of exercise training in lowering blood pressure: a meta-analysis of randomized controlled trials of 4 weeks or longer. J Hum Hypertens 1997;11:641-649.

31. Farinatti PT, Assis B. Estudo da freqüência cardíaca, pressão arterial e duplo produto em exercícios contra-resistência e aeróbio contínuo. Rev Bras Ativ Física Saúde 2000;5:5-16.

32. Gotshal RW, Gootman J, Byrnes WC, Fleck SJ, Valovich TC. Noninvasive characterization of the blood pressure response to the double-leg press exercise. J Exerc Physiol [periódico on line] 2003;9:78-84.

33. Polito MD, Simão R, Nóbrega AC, Farinatti PT. Pressão arterial, freqüência cardíaca e duplo produto em séries sucessivas do exercício de força com diferentes intervalos de recuperação. Rev Port Ciência Desp 2004;4:7-15.

34. Veloso U, Monteiro W, Farinatti P. Exercícios contínuos e fracionados provocam respostas cardiovasculares similares em idosas praticantes de ginástica? Rev Bras Med Esporte 2003;9:78-84.

35. Polito MD, Simão R, Senna GW, Farinatti PTV. Efeito hipotensivo do exercício de força realizado em intensidades diferentes e mesmo volume de trabalho. Rev Bras Med Esporte 2003;9:01-05.

36. Simão R, Fleck SJ, Polito MD, Monteiroi WD, Farinatti PTV. Effects of resistence exercise on blood pressure in normotensive individuals using differents intensities, volumes and methodologies. J Strength Cond Res, 2005 (no prelo).

37. Hill DW, Collins MA, Cureton KJ, Demello JJ. Blood pressure response after weight training exercise. J Appl Sport Sci Res1989;3:44-47.

38. Cononie CC, Graves JE, Pollock ML, Phillips MI, Sumners C, Hagberg JM. Effect of exercise training on blood pressure in 70-79 year old men and women. Med Sci Sports Exerc 1991;23:505-511.

39. O' Connor PJ, Bryant CX, Veltri JP, Gebhardt SM. State anxiety and ambulatory blood pressure following resistance exercise in females. Med Sci Sports Exerc 1993;25:516-21.

40. Fisher MM. The effect of resistance exercise on recovery blood pressure in normotensive and borderline hypertensive women. J Strength Cond Res 2001; 15:210-216.

41. Focht BC, Koltyn KF. Influence of resistance exercise of different intensities on state anxiety and blood pressure. Med Sci Sports Exerc1999;31:456-463.

42. Katz J, Wilson BRA. The effects of a six-week, low intensity nautilus circuit training program on resting blood pressure in females. J Sports Med Phys Fitness 1992;32:299-302.

43. Van Hoof, Marcor F, Lijnen P, Staessen J, Thijs L, Vanhees L, Fagard R. Effect of strength training on blood pressure measured at various conditions in sedentary men. Int J Sports Med 1996; 17:415-422.

44. Tsutsumi T. The effects of strength training on mood, self-efficacy, cardiovascular reactivity and quality of life in older adults. Boston: Boston University; 1997.

45. Kelley GA, Kelley KS. Progressive resistance exercise and resting blood pressure: a meta-analysis of randomized controlled trials. Hypertens 2000;35:838-843.

46. Pollock ML, Franklin BA, Balady GJ et al. AHA Science Advisory. Resistance exercise in individuals with and without cardiovascular disease: benefits, rationale, safety and prescription: an advisory from the Committee on Exercise, Rehabilitation, and Prevention, Council on Clinical Cardiology, American Heart Association; position paper endorsed by the American College of Sports Medicine. Circulation 2000;101:828-833.

47. Monteiro WD. Personal Training - Manual para avaliaçáo e prescrição de condicionamento físico. Rio de Janeiro: Sprint; 1999.

48. Farinatti PT, Oliveira PB, Pinto VL, Monteiro W, Francischetti E. Programa domiciliar de exercícios: efeito de curto prazo sobre a aptidão física e pressão arterial de indivíduos hipertensos. Arq Bras Cardiol 2005;88:473-479.

49. Hagberg JM, Montain SJ, Martin WH. Blood pressure and hemodynamic responses after exercise in older hypertensives. J Appl Physiol 1987;63:270-276.

50. Laughlin WH, Korthuis RJ, Duncker DJ, Bache RJ. Control of blood flow to cardiac and skeletal muscle during exercise. In: L. B. Rowell and J. T. Sheperd (Eds.). Handbook of physiology, exercise: regulation and integration of multiple systems. Bethesda: American Physiological Society; 1996. p.705-769.

51. Abboud FM. The sympathetic system in hypertension: Stateof-the-art review. Hypertension 1982;4:208-225.

52. Greenwood JP, Stoker JB, Mary DA. Single-unit sympathetic discharge: quantitative assessment in human hypertensive disease. Circulation 1999;100:1305-1310.

53. Matsukawa T, Mano T, Gotoh E, Ishil M. Elevated sympathetic nerve activity in patients with accelerated essential hypertension. J Clin Invest 1993;92:25-28.

54. Jennings G, Nelson L, Nestel P, Esler M, Korner P, Burton $\mathrm{D}$, Bazelmans J. The effects of changes in physical activity on major cardiovascular risk factors, hemodynamics, sympathetic function, and glucose utilization in man: a controlled study of four levels of activity. Circulation 1986;73:30-40.

55. Meredith IT, Jennings GL; Esler MD et al. Time-course of the antihypertensive and autonomic effects of regular endurance exercise in human subjects. J Hypertens 1990;8:859-866.

56. Urata H, Tanabe Y, Kiyonaga A et al. Antihypertensive and volume-depleting effects of mild exercise on essential hypertension. Hypertens 1987;9:245-252.

57. Chen HI, Chiang IP. Chronic exercise decreases adrenergic agonist-induced vasoconstriction in spontaneously hypertensive rats. Am J Physiol 271:H977-H983.

58. Delp MD, Mcallister RM, Laughlin M. H. Exercise training alters aortic vascular reactivity in hypothyroid rats. Am J Physiol 1996;268:H1428-H1435.

59. Spier SA, Laughlin MH, Delp MD. Effects of acute and chronic exercise on vasoconstrictor responsiveness of rat abdominal aorta. J Appl Physiol 1999; 87:1752-175.

60. Maeda S, Miyauchi T, Kakiyama T et al. Effects of exercise training of 8 weeks and detraining on plasma levels of endothelium-derived factors, endothelin- 1 a, endothelin- 1 and nitric oxid, in healthy young humans. Life Sci 2001;69:1005-16.

61. Kingwell BA Nitric Oxid-mediated metabolic regulation during exercise: effects of training in health and cardiovascular discase. FASEB J 2000;14:1685-96.

62. Anderson EA, Hoffman RP, Balon TW, Sinkey CA, Mark AL. Hiperinsulinemia produces both sympathetic neural activation and vasodilation in normal humans. J Clin Invest 1991;87:2246-2252.

63. Baron AD, Brechtel-Hook G, Johnson A, Harding D. Skeletal muscle blood flow. A possible link between insulin resistance and blood pressure. Hipertens 1993; 21:129-135. 
64. Cleroux JM, Kouamé A, Nadeau A, Coulombe D, Lacourciere Y. After effects of exercise on regional and systemic hemodynamics in hypertension. Hypertens 1992; 19:183-191.

65 . Henriksen EJ. Effects of acute exercise and exercise training on insulin resistance. J Appl Physiol 2002;93:788-796.

66. Geyssant A, Geelen G, Denis C et al. Plasma vasopressin renin activity, and aldosterone: effect of exercise and training. Eu. J Appl Physiol Occup Physiol 1981; 46:21-30.

67. Hagberg JM; Gravis JE; Limacher $M$ et al. Cardiovascular responses of 70- to 79-yr-old men and women to exercise training. J Appl Physiol 1989;66:2589-2594.

68. Nelson L, Jennings GL, Esler MD, Korner PI. Effects of changing levels of physical activity on blood-pressure and hemodynamics in essential hypertension. Lancet 1986;2:473-476.

69. Fagard R, Grauwels R, Groeseneken D, Lunen P, Staessen J, Vanhees L, Amery A. Plasma levels of rennin, angiotensin II, and 6-Ketoprostaglandin F1 alpha in endurance athletes. J Appl Physiol 1985;59:947-952.

70. Hespel P, Lunen P, Van Hoof R et al. Effects of physical endurance training on the plasma renin-angiotensin-aldosterone system in normal men. J Endocrinol 1988; 116:443-449.

71. Farinatti PTV. Aspectos da prescrição de exercício para hipertensos. Revista Brasileira de Fisiologia do Exercício; 2002.
72. Bar-Eli M. External conditions and diposition-behaviour as determinants of perceived personal causation among exercise adherence and non-adherence. Sports Science 1996;14(5):433444.

73. Biddle S, Mutrie N. Psychology of physical activity and Exercise. London: Springer-Verlag; 1991.

74. Godin G, Shepard RJ. Psychosocial factors influencing intentions to exercise in a group of individuals ranging from 45 to 74 year of age. In: Berridge M.E., Ward G R, eds. International perspectives on adapted physical activity. Champaign: Human Kinetics; 1986.

75. Smith BJ, Bauman AE, Bull FC, Bcoth ML, Harris MF. Promotion Physical activity in general practice: a controlled trial of written advice and information materials. Br J Sports Med 2000;34:262-267.

76. Cox KL, Puddey IB, Burke V, Beilin LJ, Morton AR, Bettridge HF. Determinants of change in blood pressure during S. W. E. A. T. the sedentary women exercise adherence trial. Clin Exp Pharmacol Physiol 1996;23:567-569.

77. Pinto VL, Meirelles LR, Farinatti PT. Influência de programas não formais de exercícios (doméstico e comunitário) sobre a aptidão física, pressão arterial e variáveis bioquímicas em pacientes hipertensos. Rev Bras Med Esporte 2003; 9:267-274. 